\title{
PHYSICS \\ OF SEMICONDUCTOR DEVICES
}

\section{Generation of Powerful Microwave Voltage Oscillations in a Diffused Silicon Diode}

\author{
S. K. Lyubutin ${ }^{a}$, S. N. Rukin ${ }^{a}$, B. G. Slovikovsky ${ }^{a}$, and S. N. Tsyranov ${ }^{a, b}$ \\ ${ }^{a}$ Institute of Electrophysics, Ural Branch, Russian Academy of Sciences, ul. Amundsena 106, Yekaterinburg, 620016 Russia \\ ^e-mail: rukin@iep.uran.ru \\ ${ }^{b}$ Ural Federal University, ul. Mira 19, Yekaterinburg, 620002 Russia \\ Submitted June 6, 2012; accepted for publication June 21, 2012
}

\begin{abstract}
The mechanism of the generation of powerful microwave voltage oscillations in a diffused silicon diode is studied. A reverse current $2 \mathrm{kA}$ in amplitude is passed through a $0.5-\mathrm{cm}^{2}$ diode with a structure thickness of $320 \mu \mathrm{m}$, a $p-n$ junction depth of $220 \mu \mathrm{m}$. At an average diode voltage of $\sim 300 \mathrm{~V}$ and a microwave pulse duration of $\sim 200 \mathrm{~ns}$, the maximum voltage swing reaches $480 \mathrm{~V}$. The oscillation frequency lies in the range 5 to $7 \mathrm{GHz}$; the power of the microwave pulse component is $\sim 300 \mathrm{~kW}$. A theoretical consideration shows that voltage oscillations are caused by periodically repeating processes of breakdown and structure filling with plasma followed by its removal by the reverse current. The frequency and voltage swing are controlled by the current density and dopant-concentration gradient in the vicinity of the $p-n$ junction.
\end{abstract}

DOI: $10.1134 / \mathrm{S} 1063782613050151$

\section{INTRODUCTION}

Semiconductor devices with a $p^{+}-n-n^{+}$diode structure have long been used to generate microwave voltage oscillations [1]. Most commonly used are avalanche diodes (ADs) and trapped-plasma avalanchetriggered transit (TRAPATT) diodes. The latter type is essentially an avalanche diode operating in the socalled trapped-plasma avalanche-triggered transit (TRAPATT) mode [2]. The operation mechanism of both diode types is based on the phenomenon of ionization breakdown of the semiconductor under a strong electric field.

Diode operation requires an external overvoltage pulse. In ADs, a high-frequency electric field is used to this end, which, summed with the dc bias field, provides diode breakdown in each period of external field oscillations. In the TRAPATT mode, the diode operates as a high-frequency switch whose triggering frequency is set by the frequency of the overvoltage pulses coming to the diode from an external resonator circuit. The typical microwave powers and oscillation frequencies for $\mathrm{ADs}$ are in the range from 5 (dc mode) to $50 \mathrm{~W}$ (pulsed mode) and $10-100 \mathrm{GHz}$; in the TRAPATT mode at frequencies from $600 \mathrm{MHz}$ to $10 \mathrm{GHz}$, microwave powers of up to $400 \mathrm{~W}$ per diode can be generated in the pulsed mode [3].

High-frequency oscillations can also be observed in diodes not purposely designed for these purposes. For example, in [4], relaxation microwave voltage oscillations with a swing of $\sim 600 \mathrm{~V}$ and a frequency of $\sim 0.3 \mathrm{GHz}$ were observed upon the breakdown of fast- recovery silicon drift diodes (FRDs) during the stage of reverse voltage pulse shaping. In [5], in FRDs based on GaAs structures, voltage oscillation swings of tens of volts at a frequency of $\sim 6 \mathrm{GHz}$ were achieved.

In [6], undamped microwave voltage oscillations of gigahertz frequency in a silicon $p^{+}-p-n^{+}$diode without a base were detected, which occurred at a reverse current duration of $\sim 300$ ns and a density of several $\mathrm{kA} / \mathrm{cm}^{2}$. In a diode containing four series structures, the oscillation power was estimated as $\sim 10 \mathrm{~kW}$ per $p-n$ junction. It was shown that the oscillation mechanism is not associated with external voltage pulses, and the role of the factor controlling the frequency and modulation depth of the microwave signal component is played by the reverse-current density.

The results obtained in [6] can be used not only in microwave electronics. For example, in [7], such a diode operation mode was used in a powerful nanosecond generator for eliminating prepulses across the load. The diode, connected in series to a load and containing 80 series structures $4 \mathrm{~cm}^{2}$ in area, blocked a reverse voltage prepulse $65 \mathrm{kV}$ in amplitude; as the main pulse with a sharp edge $780 \mathrm{kV}$ in amplitude was fed to the diode, it was switched to the ON state due to wave impact-ionization breakdown. The passage of a reverse current $5 \mathrm{kA}$ in amplitude through the diode for $\sim 20$ ns caused microwave voltage oscillations at a frequency of $\sim 1 \mathrm{GHz}$; in this case, a peak power of $4 \mathrm{GW}$ was switched through the diode to the load.

This work continues the study begun in [6]. In a diffused silicon diode, the generation mode of voltage oscillations at a frequency from 5 to $7 \mathrm{GHz}$ and a microwave component power of $\sim 300 \mathrm{~kW}$ is experi- 
mentally implemented. Using numerical simulation of diode processes, it is demonstrated that the oscillation frequency and swing are controlled by the current density and dopant-concentration gradient in the $p-n$ junction plane.

\section{EXPERIMENTAL}

\subsection{Experimental Scheme, Diodes under Study, and Measurement Instrumentation}

Microwave voltage oscillations in diode D under study were excited using a circuit (Fig. 1) for pumping semiconductor current interrupters based on FRD and SOS (Solid-state Opening Switch) diodes [8, 9]. Capacitors C1 and C2 (20 nF) are assembled from KVI-3 ceramic capacitors. Magnetic switches MS1 and MS2 are based on toroids made of 50 NP tape. Switch MS1 contains a ring $120 \times 60 \times 20 \mathrm{~mm}$ in size and has 7 loops; switch MS2 contains a ring $220 \times$ $120 \times 25 \mathrm{~mm}$ in size and 1 loop.

Capacitor $\mathrm{C} 1$ is charged by a power supply unit for $1 \mu$ s to a voltage of $\sim 14 \mathrm{kV}$. During this time, switch MS1 blocks the increasing voltage at $\mathrm{C} 1$. At the instant of maximum voltage at $\mathrm{C} 1$, switch MS1 is saturated, and the energy is transferred from $\mathrm{C} 1$ to $\mathrm{C} 2$. The current of this process, $1.2 \mathrm{kA}$ in amplitude and $320 \mathrm{~ns}$ in duration, flows through diode $\mathrm{D}$ in the forward direction (the negative current halfwave in Fig. 2). As the voltage at $\mathrm{C} 2$ reaches $\sim 12 \mathrm{kV}$, the core of the MS2 switch is saturated. Capacitor $\mathrm{C} 2$ is recharged via the coil of the saturated switch MS2; the current of this process $2 \mathrm{kA}$ in amplitude and $350 \mathrm{~ns}$ in duration flows through $\mathrm{D}$ in the reverse direction (positive current halfwave in Fig. 2).

When a reverse current flows from the diode structure, excess carriers injected into the diode during positive current halfwave passage are carried away. At a point in time close to the reverse current maximum, the diode begins to recover its blocking properties. In this case, the applied reverse voltage sharply increases with a characteristic time of several nanoseconds. The short edge of the appearing voltage applied to the diode initiates wave impact-ionization breakdown in it, and the subsequent reverse current flow through the diode occurs in the mode of the generation of microwave voltage oscillations. The diode resistance in both forward and reverse directions is much lower than the wave resistance of the pumping circuit operating in a mode close to that of the short-circuit mode.

The oscillogram in Fig. 2 illustrates the average reverse voltage applied to the diode without displaying the microwave signal component on the oscilloscope screen (the "Average" recording mode with 100300 overlapping pulses was used). The oscillograms of pulses displaying the microwave signal component, to be discussed below, are shown in Fig. 3. Here we only note that a microwave oscillation in the diode can be obtained even without using the circuit in Fig. 1, con-

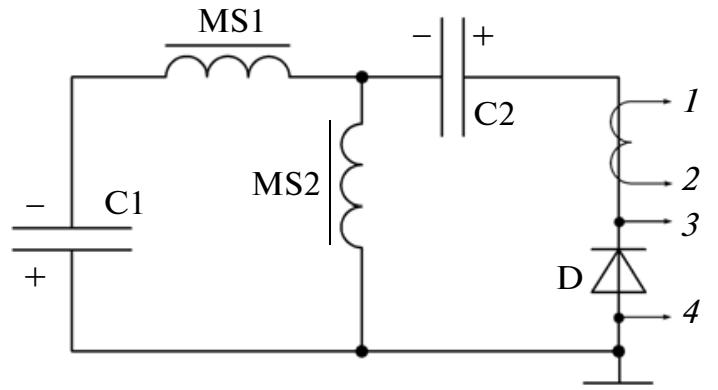

Fig. 1. Schematic of the experimental setup.

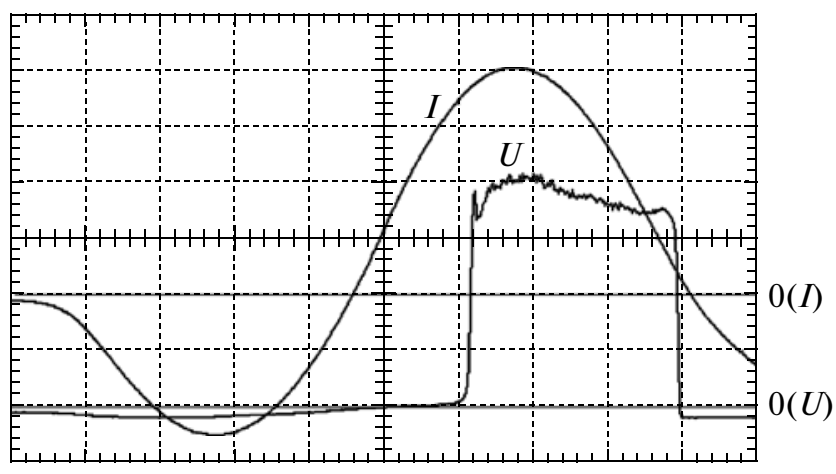

Fig. 2. Oscillograms of the diode current $(I, 500 \mathrm{~A} /$ div $)$ and average voltage $(U, 80 \mathrm{~V} / \mathrm{div})$. The time sweep is $80 \mathrm{~ns} / \mathrm{div}$. The "Average" recording mode.

taining the preliminary direct pumping unit. To this end, a reverse voltage pulse should be fed to the diode, whose edge steepness is sufficient to initiate wave impact-ionization breakdown and to provide a reverse-current density of several $\mathrm{kA} / \mathrm{cm}^{2}$ after breakdown, as was implemented in [6].

The structure of diodes $\mathrm{D}$ fabricated by the diffusion technology was $p^{+}-p-n-n^{+}$. The initial resistivity of the $n$-type silicon was $50 \Omega \mathrm{cm}$; the structure thickness was $320 \mu \mathrm{m}$. The diodes were made without a housing and without coolers and represented nickel-coated silicon wafers $5 \times 5 \mathrm{~mm}$ in size (with an area of $\left.S=0.25 \mathrm{~cm}^{2}\right)$ or $7 \times 7 \mathrm{~mm}\left(S=0.5 \mathrm{~cm}^{2}\right)$. Diodes with $p-n$ junction depths $X_{p}=160-220 \mu \mathrm{m}$ were studied.

The current pulse through the diode was measured using a Rogowski coil (points 1 and 2 in Fig. 1). The voltage from the diode $\mathrm{D}$ was directly fed to a $50-\Omega$ signal measurement circuit including a Times Microwave SFT-304 microwave cable $2 \mathrm{~m}$ long with a cutoff frequency higher than $20 \mathrm{GHz}$ and Barth Electronics high-voltage attenuators with a passband of $30 \mathrm{GHz}$.

A Tektronix TDS-6154C real-time digital oscilloscope (with a passband of $15 \mathrm{GHz}$ and a sampling count of $25 \mathrm{ps)}$ was used as a recorder. To reduce damping of the microwave signal component due to 

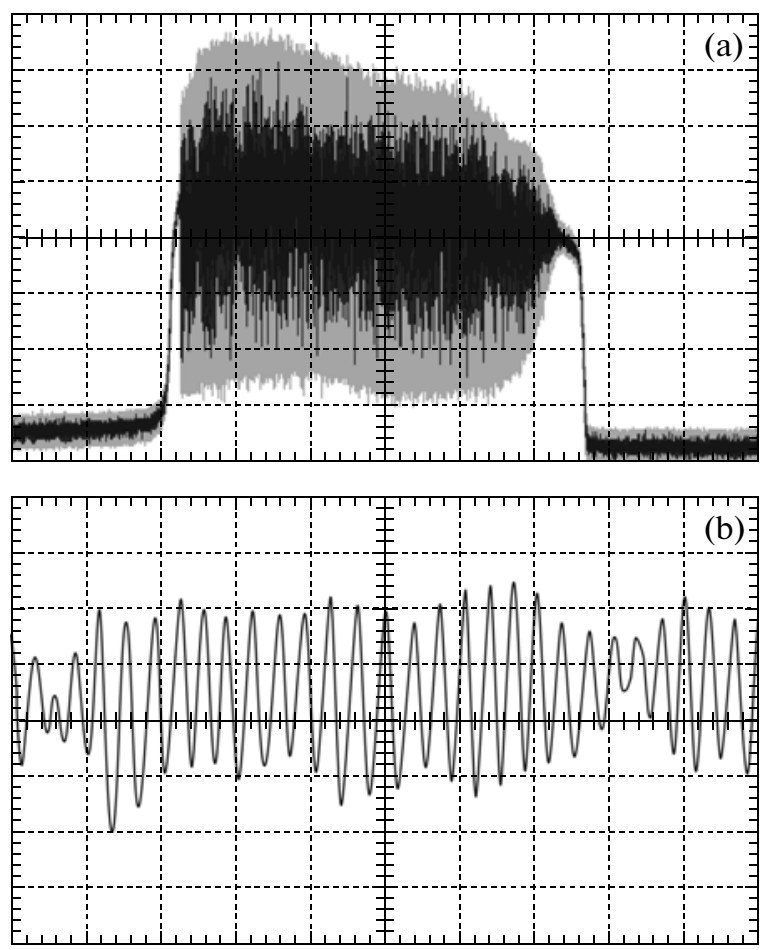

Fig. 3. Oscillograms of voltage pulses to the diode, in the "Sample" recording mode; readings every 25 ps; the amplitude scale is $80 \mathrm{~V} /$ div: (a) microwave signal component envelopes when recording a single pulse (black background) and for 2500 overlapping pulses (gray background), the time sweep is $40 \mathrm{~ns} / \mathrm{div}$; (b) the internal structure of the microwave signal component for a single pulse, the time sweep is $500 \mathrm{ps} / \mathrm{div}$.

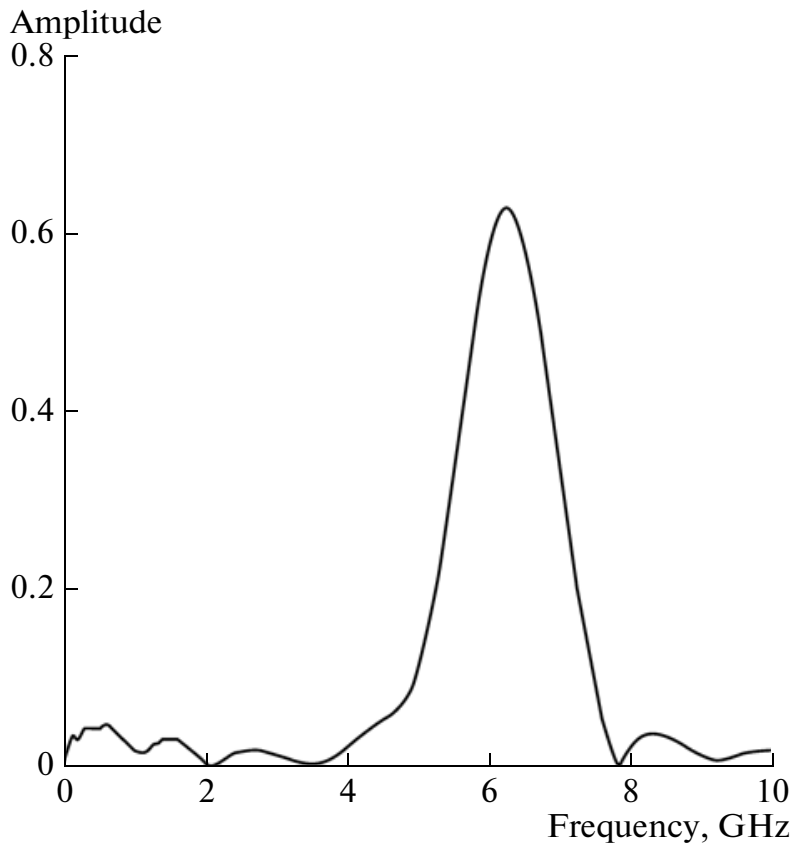

Fig. 4. Spectrum of the microwave component of the diode voltage pulse: $J=4 \mathrm{kA} / \mathrm{cm}^{2}$, the conversion interval is $5 \mathrm{~ns}$. the finite inductance of the diode connection to the measuring circuit, diode plates were soldered directly to a measuring SMA radio-frequency connector (contacts 3 and 4 in Fig. 1). Since the diodes had no protective lateral coating, the connector and the diode were placed into a dielectric cell filled with transformer oil.

\subsection{Experimental Results}

The experiments were performed at a pulse repetition rate of $0.5 \mathrm{~Hz}$. Diodes with four $X_{p}$ values, $X_{p}=$ $160,180,200$, and $220 \mu \mathrm{m}$, measured with an error of $\pm 5 \mu \mathrm{m}$ were tested. The maximum reverse-current density $J_{m}$ was varied from 2 to $8 \mathrm{kA} / \mathrm{cm}^{2}$ by installing diodes of various areas $S\left(0.25\right.$ or $\left.0.5 \mathrm{~cm}^{2}\right)$ and varying the charging voltage of capacitor C1. During the operation of diodes with $X_{p}=160$ and $180 \mu \mathrm{m}$, sparkovers on their lateral surface were observed, which resulted in device failure. Diodes with $X_{p}=200$ and $220 \mu \mathrm{m}$ operated reliably without sparkovers; the number of pulses during the measurements reached ten thousand. The best results in terms of amplitude of the generated voltage oscillations were achieved for the $0.5-\mathrm{cm}^{2}$ diode with $X_{p}=220 \mu \mathrm{m}$ at a reverse-current amplitude of $2 \mathrm{kA}\left(J_{m}=4 \mathrm{kA} / \mathrm{cm}^{2}\right)$. The diode current and voltage oscillograms shown in Figs. 2 and 3 correspond to this version.

An oscillogram showing the average diode voltage $U$ is shown in Fig. 2 . The voltage $U$ weakly varies during the pulse and is $\sim 300 \mathrm{~V}$. The oscillogram of the microwave signal component envelope is shown in Fig. 3a. The maximum diode voltage reaches $500 \mathrm{~V}$, the residual voltage is $50-100 \mathrm{~V}$, the maximum swing of observed voltage oscillations $\Delta U_{m}$ is $\sim 400 \mathrm{~V}$. The microwave-voltage pulse duration controlled by the reverse-current pulse duration is $\sim 200 \mathrm{~ns}$. We note that the finite oscilloscope-sampling frequency (counts in $25 \mathrm{ps)}$ has an effect on the measured $\Delta U_{m}$ when recording the microwave signal component envelope (Fig. 3a). When recording a single pulse, $\Delta U_{m}$ does not exceed $320 \mathrm{~V}$; in the case of 2500 overlapping pulses, $\Delta U_{m}$ reaches $480 \mathrm{~V}$. The internal structure of voltage oscillations near the current maximum is shown in Fig. 3b.

The Fourier expansion of the microwave signal component is shown in Fig. 4. The spectrum was constructed using the mathematical package of the oscilloscope, the conversion interval was 5 ns and corresponded to the pulse region, where the diode current is at a maximum $\left(J_{m}=4 \mathrm{kA} / \mathrm{cm}^{2}\right)$. For the spectrum in Fig. 4, the central oscillation frequency $f_{c}$ is $6.25 \mathrm{GHz}$, the spectrum width at the levels of 3 and $10 \mathrm{~dB}$ is $18 \%$ and $32 \%$, respectively.

Assuming that the voltage oscillations are harmonic, the maximum power $P_{m}$ of the microwave pulse component in the region with the maximum 
diode current $I_{m}$ can be estimated from the relation $P_{m}=I_{m} \Delta U_{m} k_{u} / \pi$, where $k_{u}$ is the signal attenuation factor in the measuring circuit. The signal attenuation in the measuring circuit at $f=6 \mathrm{GHz}$, taking into account damping in the measuring cable (certified value) and damping due to the finite inductance of the diode structure's connection to the connector $(\sim 0.5 \mathrm{nH})$, was $1.6 \mathrm{~dB}\left(k_{u}=1.2\right)$. Taking $I_{m}=2 \mathrm{kA}$ and $\Delta U_{m}=400 \mathrm{~V}$, we obtain a microwave component power of $P_{m} \sim 300 \mathrm{~kW}$ at the average input power to the diode in this pulse region of $600 \mathrm{~kW}\left(I_{m}=2 \mathrm{kA}, U=\right.$ $300 \mathrm{~V})$.

The generation of microwave voltage oscillations in the experimental circuit occurs in the sloping portion of the reverse current through the diode (Fig. 2). A decrease in the diode current density during the microwave pulse duration is accompanied by a decrease in the oscillation frequency $f_{c}$. The spectrum in Fig. 4 was measured in the region with the maximum diode current $I_{m} \approx 2 \mathrm{kA}$, where $f_{c}$ is at a maximum and is in the range of $6.2-6.6 \mathrm{GHz}$. The spectra measured in the following pulse regions showed that $f_{c} \approx 5.7-6.2 \mathrm{GHz}$ at $I \approx 1.7 \mathrm{kA}$ (pulse center) and $f_{c} \approx$ $4.7-5.2 \mathrm{GHz}$ at $I \approx 600$ A (near the end of the microwave pulse).

The generation of diode voltage oscillations terminates before the end of the reverse-current pulse. In this case, the reverse voltage to the diode is still maintained; however, oscillations are not excited, i.e., the steady-state breakdown mode is reached (region at the end of the pulse in Fig. 3a). The cutoff current density $J_{c}$ at which voltage oscillations are terminated was independent of the diode area and reverse-current amplitude and was in the range $J_{c} \approx 800-900 \mathrm{~A} / \mathrm{cm}^{2}$.

\section{CALCULATION}

The calculation was performed using a physicalmathematical model [9] consisting in simultaneous solution of the Kirchhoff equations describing the operation of an electrical circuit with a diode $\mathrm{D}$ and the equations of electron and hole dynamics in a diode structure. The parameters of the calculated electrical circuit corresponded to the experimental scheme (Fig. 1). The electron and hole dynamics in the diode structure was calculated by means of a fundamental system of equations consisting of the continuity equations for electrons and holes, the Poisson equations for an electric field, and equations for temperature. The volume rate of avalanche generation-recombination and the dependences of the electron and hole mobility on the electric field, temperature, electron-hole scattering, and scattering at ionized impurities were taken into account.

The experiment was modeled, whose results are shown in Figs. 2-4: $X_{p}=220 \mu \mathrm{m}, S=0.5 \mathrm{~cm}^{2}$, and $I_{m}=2 \mathrm{kA}\left(J_{m}=4 \mathrm{kA} / \mathrm{cm}^{2}\right)$. In the model structure

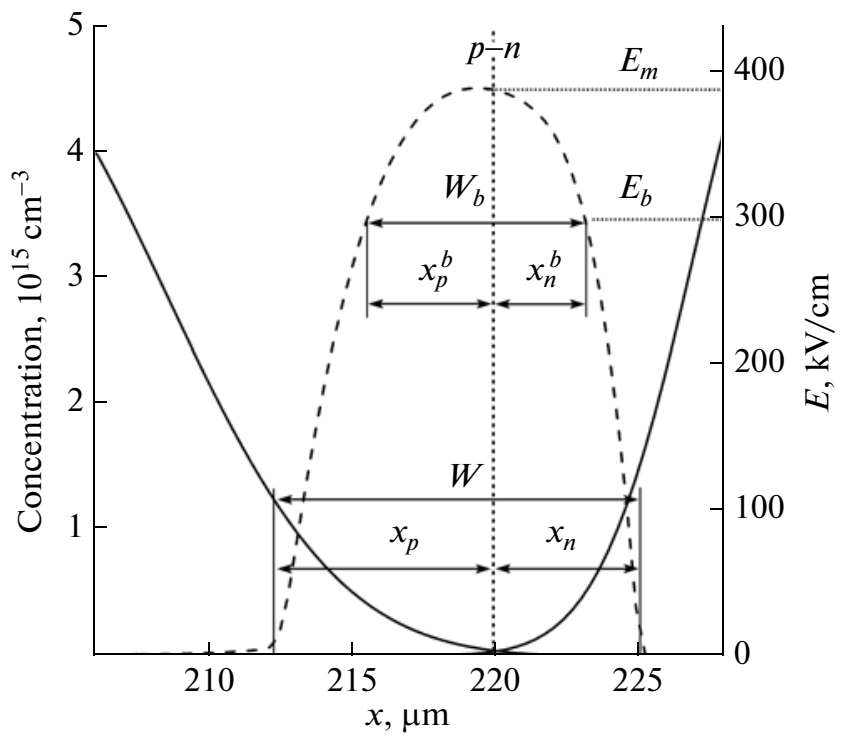

Fig. 5. Characteristic distributions of donor and acceptor concentrations (solid curves) and the electric field (dashed curve) in the vicinity of the $p-n$ junction. $E_{m}$ and $W$ are the electric-field amplitude and width of the depletion region, respectively, at the instant of the maximum diode voltage; $W_{b}$ is the width of the breakdown region according to the level $E=E_{b} \approx 300 \mathrm{kV} / \mathrm{cm}$.

$320 \mu \mathrm{m}$ thick, made of $n$-type silicon with an initial resistivity of $50 \Omega \mathrm{cm}$, dopants were distributed as follows: the $p^{+}$region was formed by boron diffusion $\left(10^{21} \mathrm{~cm}^{-3}, 80 \mu \mathrm{m}\right)$, the $p$ region was formed by aluminum diffusion $\left(10^{17} \mathrm{~cm}^{-3}, 220 \mu \mathrm{m}\right)$, and the $n^{+}$region was formed by phosphorus diffusion $\left(5 \times 10^{20} \mathrm{~cm}^{-3}\right.$, $110 \mu \mathrm{m})$. The numbers in parentheses mean the impurity boundary concentration and depth.

\subsection{Oscillation Frequency and Swing}

The calculations revealed two major factors that have the most significant effect on the voltage oscillation frequency and swing in the model diode, i.e., the diode current density and the dopant-concentration distribution in a narrow $(10-20 \mu \mathrm{m})$ layer in the vicinity of the $p-n$ junction. The time dependence of the current density and the general structure of the dopant distribution in the diode corresponded to the experimental data; various dopant-concentration distributions in the narrow layer were set by varying the concentration gradients of donors and acceptors at the point of the $p-n$ junction. In this case, two conditions were satisfied: the dopant distribution obeys Gaussian law and the dopant gradients in the $n$ - and $p$ regions are related as $\gamma_{n} / \gamma_{p} \approx\left(D_{\mathrm{Al}} / D_{P}\right)^{1 / 2}$, where $D_{\mathrm{Al}}$ and $D_{P}$ are the aluminum and phosphorus diffusion coefficients in silicon. One of the distributions is shown in Fig. 5. 


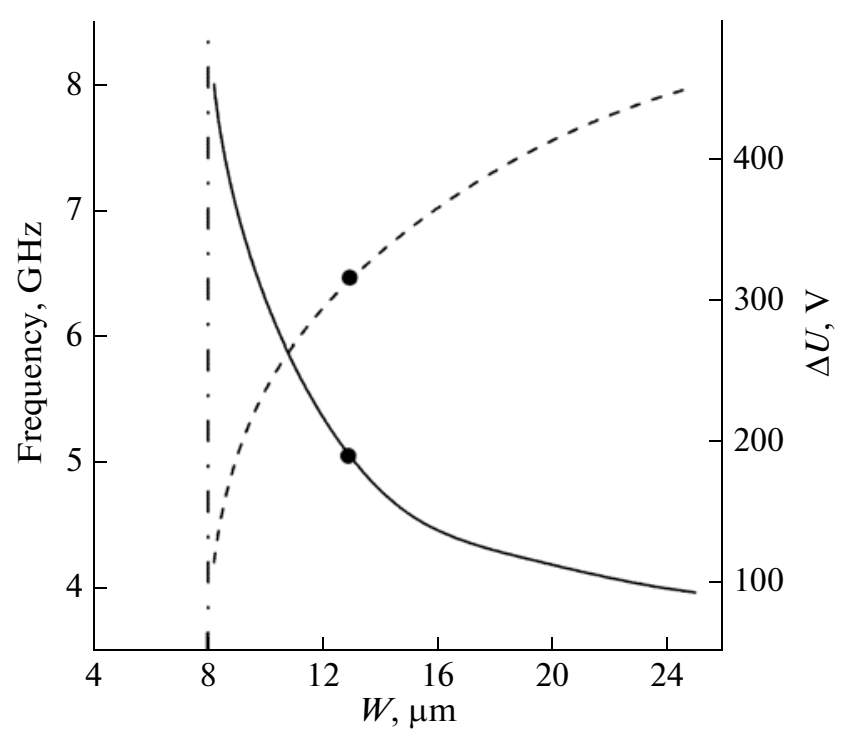

Fig. 6. Calculated dependences of the frequency (solid curve) and voltage oscillation swing (dashed curve) on the width of the depletion region $W$ at $J=4 \mathrm{kA} / \mathrm{cm}^{2}$.

The depletion width $W$ in which a high electric field is implemented vary with the dopant-concentration gradients.

A series of numerical calculations was performed for structures with various dopant-concentration gradients at the point of the $p-n$ junction. The results are shown in Fig. 6 in the form of a dependence of the oscillation frequency and swing on the depletion width $W$ at $J_{m}=4 \mathrm{kA} / \mathrm{cm}^{2}$. We can see that as $W$ decreases, which corresponds to an increase in the dopant-concentration gradient at the point of the $p-n$ junction, the oscillation frequency increases, while the oscillation swing decreases. The best agreement between the calculated and experimental data is achieved at $W \approx$ $13 \mu \mathrm{m}$ (points in Fig. 6), when $\gamma_{p}=6.5 \times 10^{18} \mathrm{~cm}^{-4}$. In this case, the disagreement between the experimental and calculated oscillation frequencies and swings does not exceed $20 \%$.

It was also found that voltage oscillations are not excited in the structure at $W$ less than $\sim 8 \mu \mathrm{m}$ (vertical dash-dotted line in Fig. 6); steady-state breakdown conditions are implemented in the vicinity of the $p-n$ junction, when the carrier-concentration and electric-field distributions do not change with time.

\subsection{Oscillation Generation}

Let us consider the process of oscillation generation in a structure with a depletion width of $W=$ $13 \mu \mathrm{m}$. The calculated time dependences of the diode current density and voltages are shown in Fig. 7a (in comparison with Fig. 1, the reverse voltage to the capacitors and the reverse connection of the diode D are used in the calculated circuit). We can see that the generation of voltage oscillations begins in the diode at
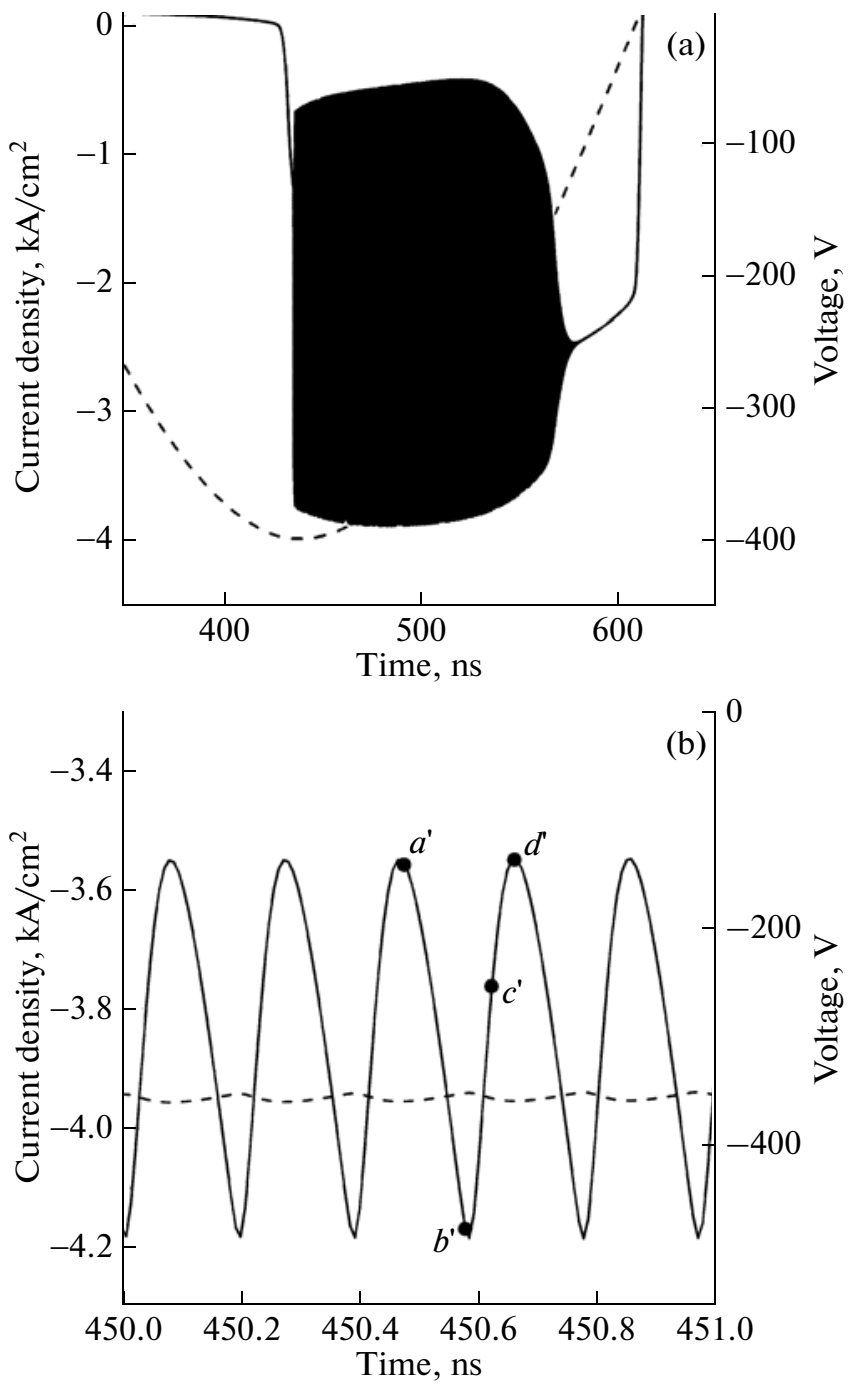

Fig. 7. Calculated time dependences of the diode current density (dashed curve) and voltage (solid curve) (a) in the entire oscillation range and (b) in the time interval of 450451 ns.

a point in time close to the reverse current maximum, which last for $\sim 150 \mathrm{~ns}$, terminating at a cutoff current density $J_{c} \approx 1 \mathrm{kA} / \mathrm{cm}^{2}$. At $J<J_{c}$, the steady-state breakdown mode is reached in the structure.

Figure $7 \mathrm{~b}$ shows the voltage oscillations in a time interval of $1 \mathrm{~ns}$. The oscillation swing $\Delta U$ reaches $320 \mathrm{~V}$ at a frequency of $5.1 \mathrm{GHz}$, which is consistent with the experimental data ( $\sim 400 \mathrm{~V}, \sim 6 \mathrm{GHz})$. To analyze the numerical simulation results, let us analytically estimate the oscillation parameters. Assuming that the dopant distribution near the $p-n$ junction is linear, the field distribution in the region of the $p-n$ junction obeys the equation

$$
\frac{d E}{d x}=\frac{e \gamma_{p, n} x}{\varepsilon}
$$


where $\varepsilon=1.0 \times 10^{-12} \mathrm{~F} / \mathrm{cm}$ is the permittivity of silicon, $\gamma_{p, n}$ is the dopant-concentration gradient in the $p$ - and $n$ regions, $x$ is the distance from the $p-n$ junction plane, and $e$ is the electron charge. From Eq. (1), we can estimate the depletion width $W$,

$$
W=c\left(\frac{2 \varepsilon E_{m}}{e \gamma_{p}}\right)^{1 / 2}
$$

where $c=1.7$ is obtained from the condition $\gamma_{p} / \gamma_{n}=0.5$.

Let us now consider the formation of voltage oscillations in the structure in the time interval between points $a^{\prime}$ and $d^{\prime}$ (Fig. 7b), which corresponds to one oscillation period. The processes considered include three stages: electric field enhancement to a maximum value, breakdown and structure filling with plasma, and plasma removal by the reverse current.
In the first stage, the electric field is low near the voltage minimum (point $a^{\prime}$ in Fig. 7b), since the donor and acceptor charge in the $p-n$-junction region is almost fully compensated by majority carriers (Fig. 8a). The reverse current through the diode removes majority carriers, and the field amplitude near the $p-n$ junction increases. When the field strength exceeds the ionization threshold $E_{b} \approx 300 \mathrm{kV} / \mathrm{cm}$, avalanche carrier multiplication begins. The charge of carriers formed during avalanche multiplication has no time to compensate for the rapidly enhanced field, and its amplitude reaches the maximum $E_{m} \approx$ 400 kV/cm (Fig. 8b).

Let us estimate the characteristic time of field enhancement to the maximum value (time interval
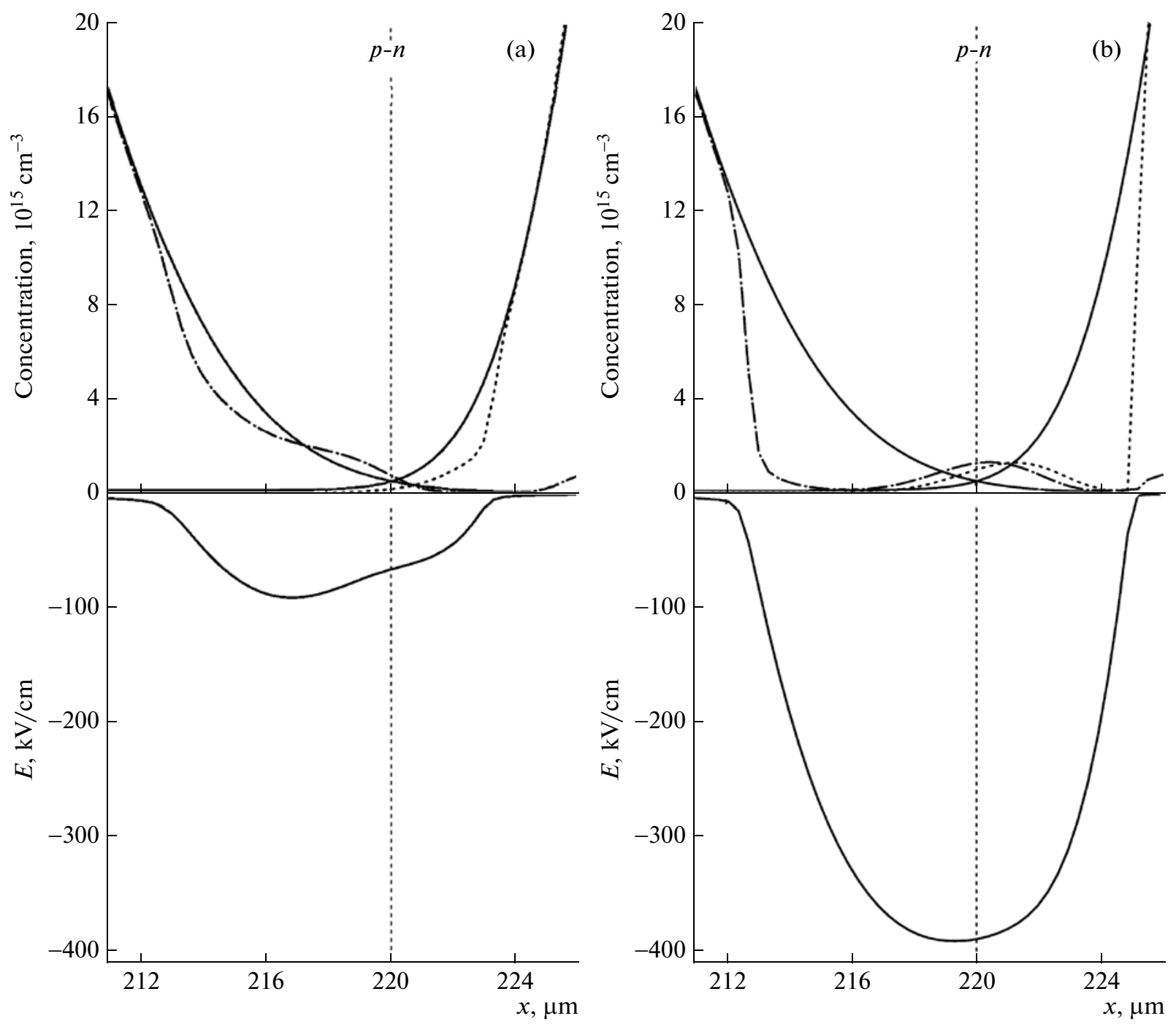

Fig. 8. Distributions of the hole (dash-dotted curve) and electron (dashed curve) concentrations and the electric field in the vicinity of the $p-n$ junction, calculated at the time points $t=$ (a) 450.47 , (b) 450.58 , and (c) $450.62 \mathrm{~ns}$ ( $a^{\prime}, b^{\prime}$, and $c^{\prime}$ in Fig. 7b). The fixed distributions of the donor and acceptor concentrations in the structure, used in the calculation are shown by solid curves. 


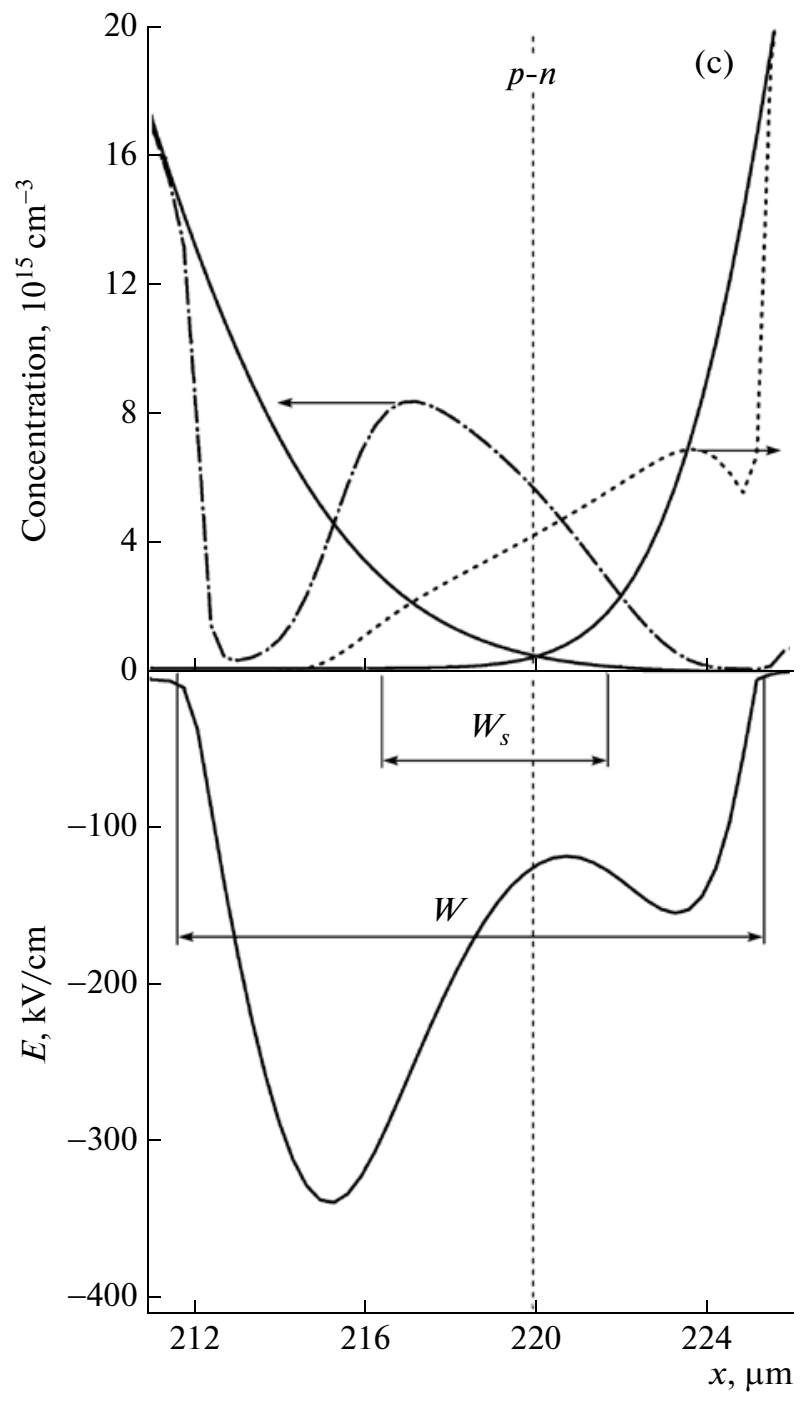

Fig. 8. (Contd.)

$a^{\prime}-b^{\prime}$ in Fig. 7b). The total current in the $p-n$-junction plane is defined by the equation

$$
j=j_{c}+j_{d}=j_{c}+\varepsilon \partial E / \partial t,
$$

where $j_{c}$ and $j_{d}$ are the conduction and bias current densities, respectively.

As the $p-n$ junction is freed of majority carriers, the contribution of $j_{c}$ to the total current continuously decreases. Neglecting the conduction current, let us estimate the time $\tau_{1}$ required to reach the field maximum $E_{m}$,

$$
\tau_{1}=\frac{\varepsilon E_{m}}{|j|} \approx 100 \mathrm{ps}
$$

The beginning of the second stage corresponds to the point $b^{\prime}$ in Fig. $7 \mathrm{~b}$.
At this point in time, an ionization wave is formed in the structure, whose front propagates on both sides of the $p-n$ junction. Assuming that the space charge near the $p-n$ junction is formed mostly by charged impurities (Fig. 8b), from Eq. (1), the electric-field distribution at the beginning of the ionization process can be written as

$$
E(x, 0)=-E_{m}+\frac{e \gamma_{p, n} x^{2}}{2 \varepsilon} .
$$

Since the current beyond the boundaries of the ionization front is the bias current, the coordinate and time dependence of the field is given by

$$
E(x, t)=E(x, 0)-\frac{j t}{\varepsilon} .
$$

The avalanche-ionization region expands in time with the wave-front velocity $V_{f}$, which can be obtained from Eqs. (5) and (6) under the condition that $E(x, t)=-E_{m}$ at the boundary of the front,

$$
V_{f}=\left(\frac{j}{2 e t \gamma_{p, n}}\right)^{1 / 2} \text {. }
$$

As the ionization region expands, the velocity $V_{f}$ decreases and, by $t_{s}$, becomes equal to the saturated carrier drift velocity in silicon $V_{s} \approx 10^{7} \mathrm{~cm} / \mathrm{s}$,

$$
t_{s}=\frac{j}{2 e V_{s}^{2} \gamma_{p, n}} \text {. }
$$

In the $p$ - and $n$ regions, the time $t_{s}$ is 19 and $9.5 \mathrm{ps}$, respectively. The width of the region in which $V_{f}>V_{s}$ is $W_{s} \approx 5.7 \mu \mathrm{m}$ (Fig. 8c), and the average velocity of the ionization front twofold exceeds the saturated one.

Thus, in the initial stage, the ionization front propagates as in the TRAPATT mode [2], when formed electrons and holes have no time to leave the rapidly expanding ionization region. In general the TRAPATT mode is implemented in the uniformly doped base region, which provides ionization-front motion with a constant velocity exceeding the saturation velocity. In the case at hand, as the ionization front moves from the $p-n$-junction plane, the dopant concentration monotonically increases, and the front velocity decreases, reaching the saturation velocity by the time $t_{s}$.

Then, outside the $W_{s}$ region, the ionization front moves with the saturation velocity, filling it with electron-hole plasma. Taking into account that $E>E_{b}$, the width of this region is given by

$$
W_{b}=c\left(\frac{2 \varepsilon\left(E_{m}-E_{b}\right)}{e \gamma_{p}}\right)^{1 / 2} .
$$

Beyond the boundary $W_{b}$, the ionization processes terminate, and the current flows due to the motion of majority carriers with the saturation velocity. Since 
carriers move with the saturation velocity and $x_{p}>x_{n}$ (see Fig. 5), the duration $\tau_{2}$ of the stage of structure filling with plasma is controlled by the time of passage of the concentration front through the $p$-type part of the depletion region $x_{p}$,

$$
\tau_{2}=\left(\frac{x_{p}}{V_{s}}\right)-t_{s} \approx 68 \mathrm{ps}
$$

Let us now consider the stage of plasma removal from the depletion region by the reverse current. This stage begins simultaneously with the beginning of ionization, i.e., the processes of structure filling with plasma and its removal from the structure occur simultaneously. The duration of the stage of plasma removal is also controlled by the more extended region $x_{p}$. The total time $\tau_{3}$ of the plasma removal stage can be defined as the sum of the time of the ionization-wave path from the $p-n$ junction to the avalanche multiplication boundary and the time of the transit of electrons produced during multiplication in the opposite direction to the $p-n$ junction. Then, under the condition $W_{b}>W_{s}$,

$$
\tau_{3}=\left(\frac{2 x_{p}^{b}}{V_{s}}\right)-t_{s} \approx 70 \mathrm{ps}
$$

where the region $x_{p}^{b}=W_{b} / c$ is shown in Fig. 5 .

After carrier removal, the electric field in the vicinity of the $p-n$ junction begins to increase and the process is repeated. As a result, undamped voltage oscillations arise in the diode. The oscillation period $\tau$ can be estimated as the sum of the durations of the stages of electric-field enhancement to a maximum $\left(\tau_{1}\right)$ and plasma removal from the breakdown region $\left(\tau_{3}\right)$,

$$
\tau=\frac{\varepsilon E_{m}}{j}+\left[2\left(\frac{2 \varepsilon\left(E_{m}-E_{b}\right)}{e \gamma_{p} V_{s}^{2}}\right)^{1 / 2}-\frac{j}{2 e \gamma_{p} V_{s}^{2}}\right] .
$$

Estimation of the oscillation period $\tau$ using Eq. (12) yields $\sim 170 \mathrm{ps}$ (frequency $f_{c} \approx 5.9 \mathrm{GHz}$ ). The first term in Eq. (12) defines the duration of the fieldenhancement stage and depends only on the density of the current passing through the structure; the second term is equal to the duration of carrier removal from the breakdown region and depends on both the current density and the dopant-concentration gradient in the vicinity of the $p-n$ junction.

\section{RESULTS AND DISCUSSION}

In the experimental section, it was found that a decrease in the current density through the diode during the microwave pulse is accompanied by a decrease in the oscillation frequency. Expression (12) yields a similar dependence, where a decrease in the current density increases both the time required for electric- field enhancement to $E_{m}$ and the time of carrier removal from the breakdown region. As a result, the oscillation period increases and the oscillation frequency decreases.

As in the numerical calculation (Fig. 6), formula (12) shows that the oscillation frequency $f_{c}$ as the dopant-concentration gradient $\gamma_{p}$ in the vicinity the $p-n$ junction increases (the depletion width $W$ decreases). We note that, at large $\gamma_{p}$ (small $W$ ), the oscillation frequency is defined by the first term, i.e., the duration $\tau_{1}$ of the field-enhancement stage; at small $\gamma_{p}$ (large $W$ ), the frequency begins to be defined by the second term, i.e., the duration $\tau_{3}$ of the plasmaremoval stage.

The characteristic times $\tau_{1} / \tau$ obtained experimentally (oscillogram in Fig. 3b), numerically (Fig. 7b), and analytically using formula (12) are $\sim 100 / 68$, $\sim 116 / 84$, and $\sim 100 / 70 \mathrm{ps}$, respectively. The values of $\tau_{1} / \tau_{3}$ were measured near the diode current maximum $\left(J_{m}=4 \mathrm{kA} / \mathrm{cm}^{2}\right)$. Substitution of the experimental values of $\tau_{1}$ and $J_{m}$ into Eq. (4) yields the maximum electric-field strength $E_{m} \approx 400 \mathrm{kV} / \mathrm{cm}$ in the structure, which corresponds to the value obtained by numerical calculation.

It should also be noted that the experimentally observed amplitude modulation of the microwave signal (Fig. 3b) is lacking in the calculations. This can be associated with the nonuniform current distribution over the diode area, whose effect is ignored within the utilized one-dimensional approximation.

An important feature of the oscillation mode under consideration is the fact that the electric field in the depletion region $W$ exceeds $E_{s} \approx 30 \mathrm{kV} / \mathrm{cm}$ (Fig. 8); therefore, carrier motion occurs with the saturation velocity $V_{s}$. This provides a significantly reduced time of the stage of plasma removal from the breakdown region in comparison with the TRAPATT mode and an increase in the frequency of generated oscillations.

Using the above characteristic values of the field and oscillation frequency, we can estimate the power of the microwave signal component. In the case at hand, the power associated with electromagnetic-field energy fluctuations in the structure is written as

$$
P=V_{E} \frac{\partial}{\partial t}\left(\frac{\varepsilon E^{2}}{2}\right) \approx\left(S W_{b}\right) \frac{\varepsilon E_{m}^{2}}{\tau},
$$

where $V_{E}$ is the structure region volume with varying electric field, $S$ is the diode area, $W_{b}$ is the width of the avalanche multiplication region from Eq. (9), and $\tau$ is the oscillation period. Estimation using Eq. (13) yields $P \approx 0.3 \mathrm{MW}$.

For comparison, the most powerful silicon TRAPATT diodes emit a power of $\sim 0.4 \mathrm{~kW}$ at a frequency of $\sim 1 \mathrm{GHz}$ [3]. A significant increase in the microwave oscillation power in the studied diode in comparison with a TRAPATT diode is first of all con- 
trolled by the larger volume $V_{E}$ (an increase by a factor of $\sim 200$ ) and the higher oscillation frequency (by a factor of $\sim 5$ ).

\section{ACKNOWLEDGMENTS}

This study was supported by the Russian Foundation for Basic Research (projects nos. 10-08-00313 and 12-08-01152) and the Presidium of the Ural Branch of the Russian Academy of Sciences (project no. 12-U-2-1014).

\section{REFERENCES}

1. S. M. Sze and R. M. Ryder, Proc. IEEE 59, 1140 (1971).

2. H. J. Prager, K. K. N. Chang, and S. Weisbrod, Proc. IEEE 55, 586 (1967).
3. A. I. Lebedev, Physics of Semiconductor Devices (Fizmatlit, Moscow, 2008), ch. 6 [in Russian].

4. V. A. Kozlov, A. F. Kardo-Sysoev, and V. I. Brylevskii, Semiconductors 35, 608 (2001).

5. V. A. Kozlov, A. V. Rozhkov, and A. F. Kardo-Sysoev, Semiconductors 37, 1428 (2003).

6. S. A. Darznek, S. K. Lyubutin, S. N. Rukin, and B. G. Slovikovskii, Semiconductors 36, 599 (2002).

7. A. I. Bushlyakov, S. K. Lyubutin, A. V. Ponomarev, S. N. Rukin, B. G. Slovikovskii, S. P. Timoshenkov, and S. N. Tsyranov, Prib. Tekh. Eksp., No. 4, 72 (2006) [Instrum. Exp. Tech. 49, 516 (2006)].

8. I. V. Grekhov and G. A. Mesyats, Phys. Usp. 48, 703 (2005).

9. S. K. Lyubutin, S. N. Rukin, B. G. Slovikovskii, and S. N. Tsyranov, Semiconductors 46, 519 (2012).

Translated by A. Kazantsev 